\title{
Remission of HIV-related naïve and high- risk Burkitt's lymphoma treated by autologous stem cell transplantation plus CART
}

Haiyan Min ${ }^{1 \dagger}$, Jianwei Yang ${ }^{2 \dagger}$, Sanbin Wang ${ }^{3}$, Pengfei Tao ${ }^{1}$, Yuqin Song ${ }^{4}$, Xiaopei Wang ${ }^{4}$, Huiqin $\mathrm{Li}^{1}$, Xinping Yang ${ }^{1}$, Xingqi Dong ${ }^{1}$, Fu-Sheng Wang ${ }^{5}$, Ming Shi ${ }^{5}$, Xicheng Wang ${ }^{1^{*}}$ and Ruonan $\mathrm{Xu}^{5^{*}}$

\begin{abstract}
A 27-year-old male with HIV-associated naive and high-risk Burkitt's lymphoma sequentially received short-term, highdose non-myeloablative chemotherapy and autologous CD34-positive stem cell transfusion in the setting of combined antiretroviral therapy (CART). Prompt hematopoietic recovery was observed after 2 weeks and clinical remission from Burkitt's lymphoma within approximately 30 months after transplantation. The HIV RNA load was inhibited persistently, and drug resistance was not observed. The CD4+ T cell count approached 323 cells/ $\mu \mathrm{L}$ in a recent follow-up study. This case suggests that the use of intensive non-myeloablative chemotherapy with transplantation, combined with antiretroviral therapy, in HIV-related naive and high-risk Burkitt's lymphoma was tolerated and safe.
\end{abstract}

Keywords: HIV, Naïve, Burkitt's lymphoma, Autologous stem cell transplantation, Antiretroviral therapy, CD4+T

\section{Background}

Antiretroviral therapy (ART) has changed the outcome of patients with human immunodeficiency virus (HIV) infection by efficiently inhibiting virus replication, recovering the cluster of CD4+ T cell count, and reducing the risk of opportunistic infections [1]. However, HIV-associated adverse events will continue to occur in some patients despite ART, especially in those patients who never achieve complete immunological reconstitution.

Burkitt's lymphoma (BL) accounts for $<2 \%$ of the total cases of lymphoma in China. BL originates from a germinal center that is closely related to $c-M y c$ rearrangement and infection with the Epstein-Barr virus (EBV). More than half of patients with BL have advanced disease when diagnosed [2]. A combination of ART with intensive chemotherapy can lead to good outcomes for BL. Furthermore, patients with HIV-associated lymphomas (HRLs)

\footnotetext{
* Correspondence: wxch62597@foxmail.com; xuruonan2004@aliyun.com †Haiyan Min and Jianwei Yang contributed equally to this work.

${ }^{1}$ Yunnan Provincial Hospital of Infectious Diseases, Kunming 650301, China ${ }^{5}$ Treatment and Research Centre for Infectious Disease, Beijing 302 Hospital, Beijing 100039, China

Full list of author information is available at the end of the article
}

have been considered candidates for allogeneic or autologous stem cell transfusion (ASCT) when the criteria for transplantation have been met [3]. Currently, patients with relapsed/persistent HRLs have achieved good outcomes, comparable with those of non-HIV-infected individuals, after transfusion of stem cells [4]. In the present study, we report the outcome for a patient with HIV-associated naive and high-risk BL who received ASCT after intensive chemotherapy plus combined antiretroviral therapy (cART).

\section{Materials and methods \\ Patient}

A 27-year-old male was admitted to our unit upon presentation of a painless mass in the right groin in April 2015. Biopsies of the lesion revealed lymphoma, and antibodies against HIV were positive. He refused to accept any treatment for the concomitant HIV infection. In the following 3 months, the mass became larger and ulcers formed on the skin. In addition, the right thigh

(C) The Author(s). 2018 Open Access This article is distributed under the terms of the Creative Commons Attribution 4.0 International License (http://creativecommons.org/licenses/by/4.0/), which permits unrestricted use, distribution, and reproduction in any medium, provided you give appropriate credit to the original author(s) and the source, provide a link to the Creative Commons license, and indicate if changes were made. The Creative Commons Public Domain Dedication waiver (http://creativecommons.org/publicdomain/zero/1.0/) applies to the data made available in this article, unless otherwise stated. 
also became involved. He suffered from recurrent fever, with a body temperature fluctuating from 38 to $40.5^{\circ} \mathrm{C}$, and his body weight decreased by approximately $12 \mathrm{~kg}$ within 3 months.

In August 2015, a biopsy of the mass aspirate showed $\mathrm{BL}$, and the immunohistochemical results were positive for CD20 and EBV-encoded RNA (EBER)1/2. In addition, a bone marrow biopsy showed the total chromosomes to be normal, whereas the percentage of unidentified cells was $1.8 \%$.

Using positron emission tomography-computed tomography (PET-CT), we found increased abnormal metabolism of fludeoxyglucose (FDG) in the right groin; the region had dimensions (in $\mathrm{cm}$ ) of $12.0 \times 16.5 \times 27.0$, and the boundaries were not clear. The right thigh, anterior to the bilateral mandible, neck, axillary, retroperitoneal vessel, right iliac fossa, pelvic wall, and right inguinal lymph nodes shown increased metabolism of FDG. A blood count showed abnormal levels of lactate dehydrogenase (LDH; $1579 \mathrm{U} / \mathrm{L}$ ) as well as a white blood cell (WBC) count of $4.42 \times 10^{9} / \mathrm{L}$, a neutrophil count of $2.92 \times 10^{9} / \mathrm{L}$, a hemoglobin level of $122 \mathrm{~g} / \mathrm{L}$, and platelet count of $330 \times 10^{9} / \mathrm{L}$. The patient was diagnosed as having stage IV BL.

The HIV RNA load was 51,386 copies/mL, and the CD4+ T cell count was 107 cells/ $\mu \mathrm{L}$ at the time of $\mathrm{BL}$ diagnosis. In addition, the patient was co-infected with $\mathrm{EBV}$, and the EBV DNA load was $4.09 \times 10^{4}$ copies $/ \mathrm{mL}$. No other serious opportunistic infections were present, and the CD4+ T cell count was $<200$ cells $/ \mu \mathrm{L}$; therefore, he was started on CART immediately with lamivudine (300 mg daily), tenofovir ( $300 \mathrm{mg}$ daily), and efavirenz (600 mg daily) on the 25th of August 2015. These medications were not changed, and drug resistance did not occur.

The patient was started on a standard dose of rituximab, piraubicin, vincristine, etoposide, cyclophosphamide, and prednisone acetate (R-EPOCH) chemotherapy on the 31st of August 2015 (day 0). The patient developed a fever on the 6th of September because of a Staphylococcus aureus infection in his ulcer, based on a drug-sensitivity test. The patient is allergic to penicillin; therefore, he received lincomycin ( $400 \mathrm{mg}$, q.d.s.). Local debridement was performed every day, and his temperature returned to normal 7 days after treatment. Lincomycin was stopped on day 16 because of a continuous negative result in blood culture. He was diagnosed with agranulemia (neutrophil count = $0.01 \times 10^{9} / \mathrm{L}$ ) on day 13 , and granulocyte-colony stimulating factor (G-CSF; $150 \mu$ g, q.d.s) was given until the neutrophil count reached $0.5 \times 10^{9} / \mathrm{L}$. Meanwhile, he suffered a pulmonary infection and meropenem $(0.5 \mathrm{~g}$, b.d.s.) was added until the symptoms were controlled completely (achieved on day 30). A bone marrow biopsy showed that proliferation of the bone marrow was active, and abnormal cells were not detected.
The second cycle of chemotherapy comprised rituximab, methotrexate, and cytosine arabinoside (Ara-C). It was started on the 21st of September (day 22) and stopped on the 23rd of September (day 24). No serious complications occurred, and the EBV DNA load was reduced to $5 \times 10^{3}$ copies $/ \mathrm{mL}$ on day 30 .

From the 9th of October (day 40) to the 23rd of October (day 54), he received a third cycle of chemotherapy with rituximab, cyclophosphamide, vinorelbine, pirarubicin, and dexamethasone ( + HyperCVAD). The EBV DNA load was undetectable after the third cycle of chemotherapy.

From the 30th of October (day 61) to the 1st of November (day 63), a fourth cycle of chemotherapy (rituximab plus methotrexate/Ara-C (R-MA)) was undertaken. In addition, six intrathecal injections of methotrexate, Ara- $\mathrm{C}$, and dexamethasone were used during the second and fourth cycles of chemotherapy. After the fourth cycle of chemotherapy, the HIV RNA load was $<40$ copies $/ \mathrm{mL}$, the CD4+ T cell count was 193 cells $/ \mu \mathrm{L}$, and the $\mathrm{LDH}$ level was $91 \mathrm{U} / \mathrm{L}$. PET-CT showed that the tumor volume in the right groin and the maximum standardized uptake value (SUVmax) were reduced significantly, which suggested that tumor activity had been inhibited (Fig. 1a).

During the second and fourth cycles of chemotherapy, when the neutrophil count was $<0.5 \times 10^{9} / \mathrm{L}$, G-CSF $(150 \mu$ g, q.d.s.) was administered until the neutrophil count reached $0.5 \times 10^{9} / \mathrm{L}$. Symptoms of myelosuppression were treated by infusion of packed red blood cells (RBCs) and concentrated platelets, and oral mucositis was treated by gargling with chlorhexidine. The details of the chemotherapy regimens are summarized in Fig. 2.

\section{Apheresis and processing of peripheral blood stem cells (PBSCs)}

Before stem cell mobilization, the R-EPOCH regimen was used between the 20th of November (day 113) and the 25th of November (day 118) 2015 (Fig. 2). On the 27th of November, the WBC count was $0.5 \times 10^{9} / \mathrm{L}$, and G-CSF $(5 \mu \mathrm{g} / \mathrm{kg}$ per day) was used for PBSC mobilization for the next 5 days. When the WBC count reached $4 \times 10^{9} / \mathrm{L}$ on the 2nd of December (day 125), PBSC apheresis was undertaken using a continuous blood-cell separator (COBE ${ }^{\bullet}$ Spectra; Terumo BCT, Lakewood, CO, USA). Then, CD34-positive cells were analyzed using a FACS$\mathrm{can}^{\mathrm{Tm}}$ flow cytometer (Becton Dickinson, Franklin Lakes, NJ, USA). A total of $1.03 \times 10^{6} / \mathrm{kg}$ CD34-positive cells were obtained after undertaking apheresis twice on two consecutive days. PBSC products were combined with a cytoprotectant containing a final concentration of $10 \%$ dimethylsulfoxide (Research Industries, Salt Lake City, UT, USA) and freezing at $-150^{\circ} \mathrm{C}$ for transfusion. 
a
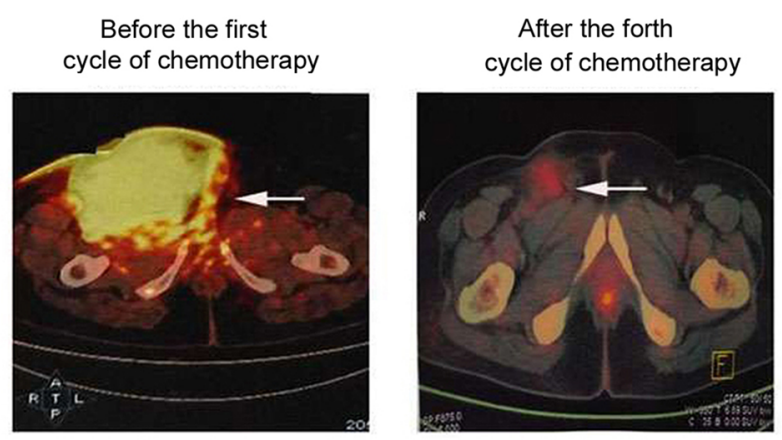

b

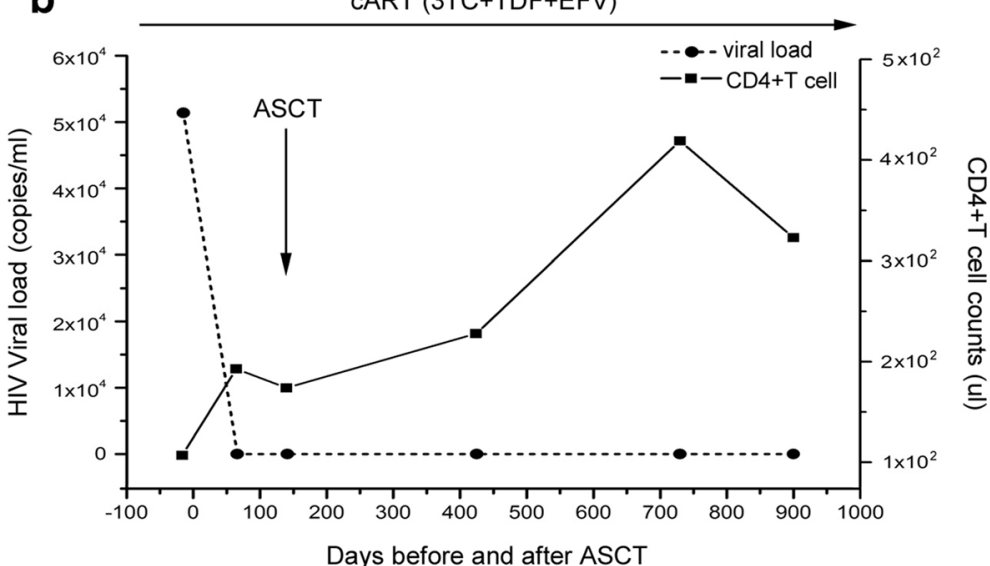

Fig. 1 a Tumor regression in the right groin after chemotherapy. Tumor evaluation by positron emission tomography-computed tomography is shown before the first cycle and after the fourth cycle of R-EPOCH. $\mathbf{b}$ HIV RNA load in plasma and CD4+ T cell count before and after autologous bone marrow transplantation. Continuous lines show the viral load and CD4+ T cell counts measured using a standard clinical test before and after autologous stem cell transplantation. 3TC, lamivudine; TDF, tenofovir; EFV, efaviren; R-EPOCH, rituximab, etoposide phosphate, prednisone, vincristine sulfate (Oncovin), cyclophosphamide, doxorubicin hydrochloride (hydroxydaunorubicin)

\section{Conditioning regimen and PBSC transfusion}

The conditioning regimen consisted of carmustine, etoposide, Ara-C, and melphalan, which began on the 11th of January (day 164) and stopped on the 16th of January 2016 (day 169). During PBSC transfusion, only myelosuppression occurred, and serious infections were not observed. Neutropenia was treated by G-CSF $(1.5 \mu \mathrm{g} / \mathrm{kg}$ per day), and symptoms of anemia and thrombocytopenia were treated by infusion of packed RBCs and concentrated platelets. Mobilized PBSCs were infused 2 days after completion of melphalan treatment on the 18th of January (day 171) and the 19th of January (day 172).

\section{Results}

\section{Remission status}

After the fourth cycle of chemotherapy, PET-CT scans of the bilateral mandible and neck, chest, abdomen, spine, and big vessels in the para-retroperitoneal, pelvic bones, and bilateral upper femur showed that multiple FDG metabolic abnormalities had disappeared. A follow-up study using bone marrow biopsies showed no morphological evidence of lymphoma, and laboratory studies did not show abnormal blood parameters. The patient remains in complete remission from his lymphoma 30 months after transplantation. In addition, after the third cycle of chemotherapy, EBV DNA was undetectable, and the EBV infection has never recurred in the follow-up study.

\section{Engraftment}

The patient reached a total neutrophil count of $0.5 \times 10^{9} / \mathrm{L}$ at 14 days and a platelet count $>25 \times 10^{9} / \mathrm{L}$ without further infusion of platelets at 25 days after ASCT. A bone marrow biopsy showed active proliferation of nucleated cells, as well as recovery of normal hematopoiesis of the bone marrow at 30 days after ASCT.

\section{HIV viral load and CD4+ T cell count}

The patient has remained in complete remission from BL for 30 months after ASCT. The HIV RNA load was $<40$ copies $/ \mathrm{mL}$ after the fourth cycle of chemotherapy, and the CD4+ T cell count was 323 cells $/ \mu \mathrm{L}$ in August 2018, as shown in Fig. 1b. 


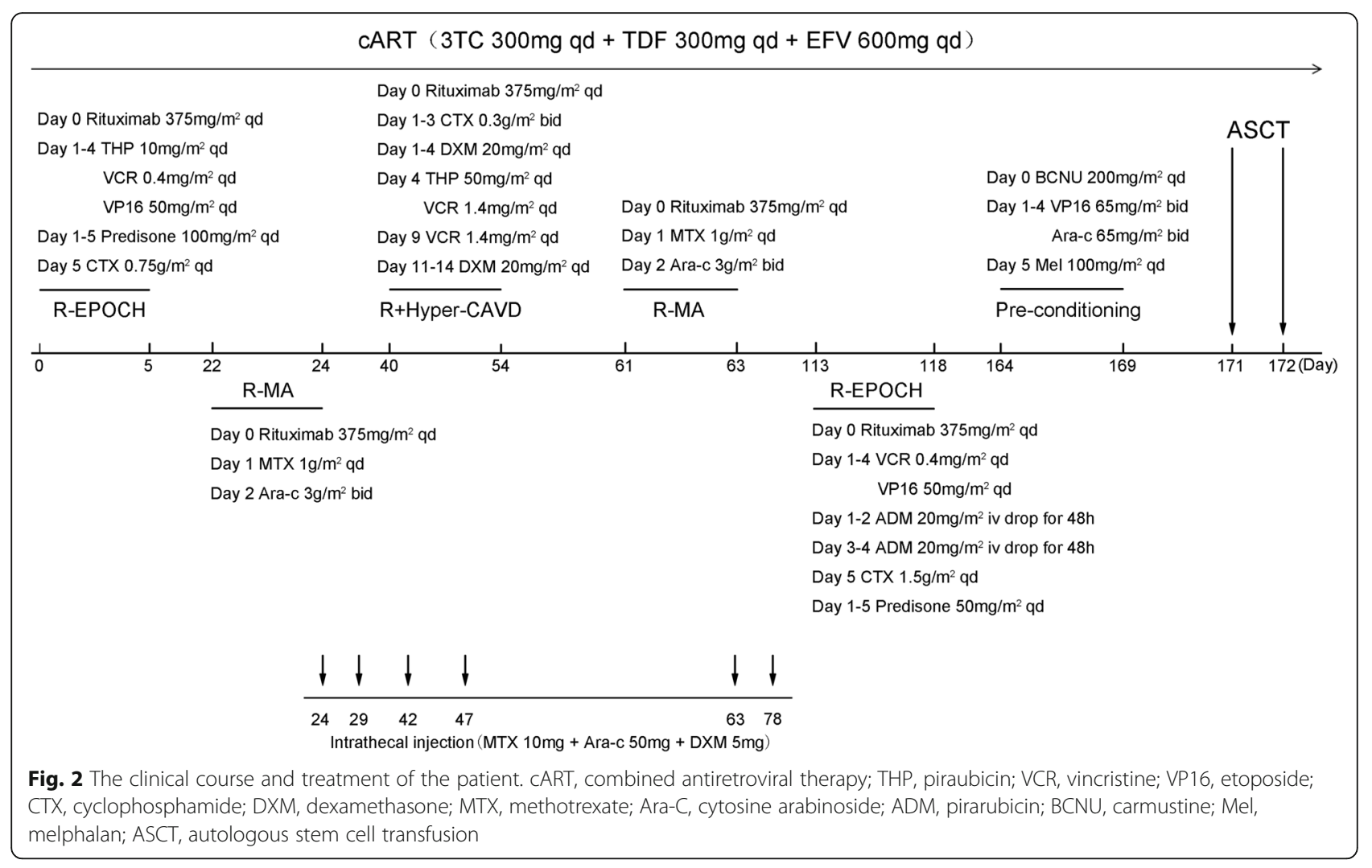

\section{Discussion}

With the development of cART, the clinical outcome and life expectancy of HIV-infected patients have improved. Nevertheless, some patients with a low CD4+ T cell count $(<200$ cells $/ \mu \mathrm{L})$ have a greater probability of acquiring HIV-associated and non-HIV-associated events [5]. The risk of developing HIV-related lymphoma in patients with AIDS is much higher than that in patients without AIDS [6].

Although studies have shown that chemotherapy and cART strategies are feasible and tolerated in patients with HIV $[7,8]$, however, it has been suggested that the use of cART plus chemotherapy should be considered carefully because of the emergence of treatment-related anemia, neurotoxicity, and mucositis; hence, there has been very limited enthusiasm for the use of high-dose chemotherapy plus cART for patients with HIV-associated lymphoma. In our study, cART was started immediately after diagnosis and throughout the course of treatment, viral duplication was well controlled, and viral rebound and drug resistance did not occur. In addition, both the standard chemotherapeutic approaches and chemoimmunotherapy were used and well tolerated.

For patients with HIV-related lymphoma, different doses of chemotherapy have been adopted considering their cytotoxicity and safety. Low and standard doses of chemotherapy carry a similar incidence of adverse effects; however, the prevalence of complete remission is
$30 \%$ and $48 \%$, respectively [9], which suggests that a standard dose of chemotherapy should be used. Apart from the use of a standard dose of chemotherapy, short-term, high-intensity chemotherapy combined with central prevention has also been reported to improve significantly the survival of HIV patients with high-risk BL [10], which suggests that active chemotherapy may benefit patients with HIV-related lymphoma.

Meanwhile, the availability of rituximab has changed treatment outcomes for patients with BL or B cell lymphoma. Particularly in patients with HIV-associated CD20-positive non-Hodgkin lymphoma, rituximab plus concurrent $\mathrm{EPOCH}$ chemotherapy is highly effective [11]. Four cycles of rituximab with $\mathrm{EPOCH}$ and hyper-CVAD regimens were used in our chemotherapy regimen, and one cycle of rituximab was used for conditioning. This strategy did not lead to serious treatment-related toxicities during chemotherapy or subsequent ASCT. More importantly, concomitant chemoimmunotherapy with cART was never stopped because of drug interactions. These results suggested that molecular-targeted drugs combined with cART were safe and well tolerated.

Sequential therapy combined with ASCT complementation was superior to single-agent chemotherapy for the treatment of malignant lymphoma [12]. However, for HIV-positive patients, hematopoietic stem cells and progenitor cells have deficiencies with regard to 
erythropoiesis, myelopoiesis, and lymphopoiesis, and mobilization using G-CSF may be limited in specific cell populations. In addition, hematopoietic stem cells can be infected by HIV-1 and might become a latent reservoir of HIV [13]. Hence, the choice of ASCT or allogeneic hematopoietic cell transplantation is a major challenge. Considering the high risk of mortality and graft-versus-host-disease response in allogeneic hematopoietic cell transplantation, after obtaining written informed consent, we chose ASCT. We made this choice because there was no obvious bone marrow involvement after chemotherapy. The recovery time of neutrophils and platelets were 14 days and 25 days, respectively, which were similar to those in non-AIDS patients. In addition, we found after three cycles of chemotherapy, the EBV load was significantly reduced, without specific anti-EBV treatment; therefore, recovery of immunological function by cART and efficient chemoimmunotherapy might be associated with the reconstruction of the anti-EBV response. Although the sustained disappearance of EBV was regarded as a good prognostic marker for EBV-positive diffuse large B cell lymphoma [14], the reason for the good control of EBV in our patient requires further study.

Recently, two HIV patients with NHL at the time of the first remission and recurrence showed good tolerance to ASCT after a transplantation-conditioning regimen comprising a high dose of cyclophosphamide, carmustine, and etoposide [4]. Those results were confirmed by a large-scale study that suggested that patients with HIV should not be denied ASCT if they meet the standard criteria for transplantation [12]. However, until now, the efficacy of short-term high-intensity chemoimmunotherapy combined with ASCT has not been identified in patients with naïve or high-risk BL when accepting concomitant cART.

Here, we reported an HIV patient with naïve and high-risk BL who accepted short-term, high-dose chemotherapy followed by ASCT. cART was not stopped because of chemotherapy-related cytotoxicity, viral resistance did not occur, and complications were well controlled by effective symptomatic treatment. Given the poor outcome of HIV-related initial and high-risk Burkitt's lymphoma, combined conventional chemotherapy with ASCT should be considered.

\section{Abbreviations \\ Ara-C: Cytosine arabinoside; ART: Antiretroviral therapy; ASCT: Autologous stem cell transfusion; BL: Burkitt's lymphoma; CART: Combined antiretroviral therapy; FDG: Fludeoxyglucose; G-CSF: Granulocyte-colony stimulating factor; HIV: Human immunodeficiency virus; HRLs: HIV-associated lymphomas; HSCT: Hematopoietic stem cell transplantation; LDH: Lactate dehydrogenase; PBSCs: Peripheral blood stem cells; PET-CT: Positron emission tomography- computed tomography; RBCs: Red blood cells; WBC: White blood cell}

\section{Acknowledgements}

We thank the patient who participated in this study for his cooperation. In addition, we would like to thank the native English-speaking scientists of Elixigen Company (Huntington Beach, California) for editing our manuscript.

\section{Funding}

This work was supported by the Capital Characteristic Clinic Project [grant number Z161100000516011].

\section{Availability of data and materials}

The datasets used during this study are available from the corresponding author on reasonable request.

\section{Authors' contributions}

HYM, JWY, SBW, FPT, and QHL were responsible for the clinical management of the patient. YQS and XPW managed the HSCT for the patient. XPY, XQD, and MS revised the manuscript. CXW, F-SW, and RNX provided research funding. All authors read and approved the final manuscript.

\section{Ethics approval and consent to participate}

This study was approved by the Ethics Committee of Peking University, China. Written informed consent was obtained from our patients before hematopoietic stem cell transplantation (HSCT).

\section{Consent for publication}

Not applicable.

\section{Competing interests}

The authors declare that they have no competing interests.

\section{Publisher's Note}

Springer Nature remains neutral with regard to jurisdictional claims in published maps and institutional affiliations.

\section{Author details}

${ }^{1}$ Yunnan Provincial Hospital of Infectious Diseases, Kunming 650301, China. ${ }^{2}$ The Third People's Hospital of Yunnan Province, Kunming 650011, China. ${ }^{3}$ Kunming General Hospital of Chengdu Military Region, Kunming 650118, China. ${ }^{4}$ Beijing Cancer Hospital, Peking University, Beijing 100142, China.

${ }^{5}$ Treatment and Research Centre for Infectious Disease, Beijing 302 Hospital, Beijing 100039, China.

Received: 2 April 2018 Revised: 9 November 2018

Accepted: 22 November 2018 Published online: 20 December 2018

\section{References}

1. Mellors JW, Rinaldo CR Jr, Gupta P, White RM, Todd JA, Kingsley LA. Prognosis in HIV-1 infection predicted by the quantity of virus in plasma. Science. 1996;272:1167-70

2. Chen Y, Dave BJ, Zhu X, Chan WC, lqbal J, Sanger WG, et al. Differences in the cytogenetic alteration profiles of diffuse large B-cell lymphoma among Chinese and American patients. Cancer Genet. 2013; 206:183-90.

3. Rodrigo JA, Hicks LK, Cheung MC, Song KW, Ezzat H, Leger CS, et al. HIVassociated Burkitt lymphoma: good efficacy and tolerance of intensive chemotherapy including CODOX-M/IVAC with or without rituximab in the HAART Era. Adv Hematol. 2012;2012:735392.

4. Bayraktar UD, Ramos JC, Petrich A, Gupta N, Lensing S, Moore PC, et al. Outcome of patients with relapsed/refractory acquired immune deficiency syndrome-related lymphoma diagnosed 1999-2008 and treated with curative intent in the AIDS Malignancy Consortium. Leuk Lymphoma. 2012; 53:2383-9.

5. Carella AM, Cavaliere M, Lerma E, Ferrara R, Tedeschi L, Romanelli A, et al. Autografting followed by nonmyeloablative immunosuppressive chemotherapy and allogeneic peripheral-blood hematopoietic stem-cell transplantation as treatment of resistant Hodgkin's disease and nonHodgkin's lymphoma. J Clin Oncol. 2000;18:3918-24.

6. Baker JV, Peng G, Rapkin J, Silverberg MJ, MacArthur RD, Cavert WP, et al. Terry Beirn Community Programs for Clinical Research on AIDS (CPCRA).CD4 + count and risk of non-AIDS diseases following initial treatment for HIV infection. AIDS 2008:22:841-848.

7. Venturelli S, Pria AD, Stegmann K, Smith P, Bower M. The exclusion of people living with HIV $(\mathrm{PLWH})$ from clinical trials in lymphoma. $\mathrm{Br} J$ Cancer. 2015;113:861-3.

8. Dunleavy K, Wilson WH. How I treat HIV associate lymphoma. Blood. 2012; 119:3245-55. 
9. Sparano JA, Wiernik PH, Hu X, Schwartz EL, Soeiro R, Henry DH, et al. Pilot trial of infusional cyclophosphamide, doxorubicin, and etoposide plus didanosine and filgrastim in patients with human immunodeficiency virusassociated non-Hodgkin's lymphoma. J Clin Oncol. 1996;14:3026-35.

10. Ratner L, Lee J, Tang S, Redden D, Hamzeh F, Herndier B, et al.

Chemotherapy for human immunodeficiency virus-associated non-

Hodgkin's lymphoma in combination with highly active antiretroviral therapy. J Clin Oncol. 2001;19:2171-8.

11. Re A, Cattaneo C, Michieli M, Casari S, Spina M, Rupolo M, et al. High-dose therapy and autologous peripheral-blood stem-cell transplantation as salvage treatment for HIV-associated lymphoma in patients receiving highly active antiretroviral therapy. J Clin Oncol. 2003;21:4423-7.

12. Alvarnas JC, Le Rademacher J, Wang Y, Little RF, Akpek G, Ayala E, et al. Autologous hematopoietic cell transplantation for HIV-associated lymphoma: results of the BMT CTN 0803/AMC 071 trial. Blood. 2016; 128:1050-8

13. van Rhee F, Giralt S, Barlogie B. The future of autologous stem cell transplantation in myeloma. Blood. 2014;124:328-33.

14. Castillo JJ, Beltran BE, Miranda RN, Young KH, Chavez JC, Sotomayor EM. EBV-positive diffuse large B-cell lymphoma, not otherwise specified: 2018 update on diagnosis, risk-stratification and management. Am J Hematol. 2018:93:953-62.

Ready to submit your research? Choose BMC and benefit from:

- fast, convenient online submission

- thorough peer review by experienced researchers in your field

- rapid publication on acceptance

- support for research data, including large and complex data types

- gold Open Access which fosters wider collaboration and increased citations

- maximum visibility for your research: over $100 \mathrm{M}$ website views per year

At BMC, research is always in progress.

Learn more biomedcentral.com/submissions 